\title{
Cultural Similarities and Differences in Social Inference: Evidence From Behavioral Predictions and Lay Theories of Behavior
}

\author{
Ara Norenzayan \\ University of Illinois, Urbana-Champaign \\ Incheol Choi \\ Seoul National University, Korea \\ Richard E. Nisbett \\ University of Michigan
}

(Kitayama \& Masuda, 2000) and Australians with Japanese (Kashima, Siegel, Tanaka, \& Kashima, 1992). Choi et al. (1999) recently reviewed the evidence for cultural differences in situational attributions.

\section{Predicting Behavior}

Everyday social judgment involves predicting the behavior of other people as much as it involves explaining their behavior or attitudes. In fact, attribution theorists have always believed that people engage in causal explanations of social events primarily to predict similar events in the future (Heider, 1958; Jones, 1979). This is apparent when, for example, one wonders if a friend will like the movie recommended to her or when a military leader trusts that an ally will support him in an upcoming battle. Past research has shown that lay dispositionism is as prevalent in prediction as it is in explanation. American participants overestimate the predictive power of

\footnotetext{
Authors' Note: This research was supported by the Russell Sage Foundation and the Office of the Vice President for Research of the University of Michigan. Part of this research was conducted while the first author was a National Science Foundation Predoctoral Fellow and the second author was a fellow of the Korean Foundation for Advanced Studies. We thank Andrea Patalano, Kaiping Peng, Jeffrey SanchezBurks, and Norbert Schwarz for their helpful comments on an earlier version of this article. We also thank Geung-Ho Cho, Jinnam Choi, Jason Drwal, Beom Jun Kim, Carolyn Nguyen, Kaiping Peng, and Mehyun Song for their assistance in conducting this research. Address correspondence to Ara Norenzayan, Department of Psychology, 603 E. Daniel Street, University of Illinois, Champaign, IL 61821; e-mail: norenzay@uiuc.edu.
}

PSPB, Vol. 28 No. 1, January 2002 109-120

C 2002 by the Society for Personality and Social Psychology, Inc. 
dispositions just as they overattribute behavior to them (Dunning, Griffin, Milojkovic, \& Ross, 1990; Kunda \& Nisbett, 1986; Newman, 1996; Ross \& Nisbett, 1991).

Surprisingly, however, most of the previous cross-cultural work in social inference-as extensive as it is-has focused primarily on explanations of behaviors and attitudes. There has been little research examining behavioral predictions across cultures. Given the centrality of behavioral prediction in everyday life, an important question is whether the cultural difference also is found for predictions of behavior. More important, comparing behavioral prediction across cultures provides one way to specify the exact psychological source of the cultural difference in social inference-whether it lies in dispositional inference, situational inference, or in both. In past cross-cultural work, it has been implicitly assumed that social inference is a unitary process. Evidence for strong situational attribution has been taken to indicate weak dispositional attributions as well. But the social inference process consists of at least two distinct cognitive operations: dispositional inference and situational inference (Gilbert \& Malone, 1995; Krull, 1993). Dispositional inference is attributing behavior to internal and enduring qualities of the individual, for example, personality and temperament. Situational inference is attributing behavior to external factors impinging on the individual, such as situations, roles, or the larger social context.

\section{Specifying the Attributional Origins of the Cultural Difference}

There are two possibilities concerning how Western and Eastern cultures might differ in behavioral predictions. If Eastern cultures have weak beliefs in dispositions, then their predictions about behavior should show low levels of cross-situational consistency. The ethnographic and cross-cultural literature certainly provides some basis for this possibility. Anthropologists have occasionally noted that dispositional thinking is rare, absent, or unimportant in non-Western societies (Hirschfeld, 1995).

There is growing evidence, however, that even members of Eastern cultures make robust dispositional inferences (Choi et al., 1999). The second possibility for cultural variation in behavioral prediction, then, is that Eastern and Western cultures make dispositional inferences in much the same way but differ in the degree to which they make situational inferences. For example, cross-cultural studies suggest that East Asians organize personality information in remarkably similar ways as Westerners, both with imported as well as indigenous instruments (e.g., McCrae, Costa, \& Yik, 1996; see Choi et al., 1999). This evidence supports the case for East Asian dispositionism to the extent that perceptions of personality structure can be taken as evidence for making dispositional inferences.

More direct social psychological evidence has recently emerged that bolsters the argument that Easterners and Westerners sometimes make dispositional inferences in much the same way. Experimental work by several researchers suggests that when situational constraints are not salient, East Asians, including Chinese, Korean, and Japanese, show the correspondence bias at least in one paradigm - the Jones and Harris (1967) attitude attribution experiments (Choi \& Nisbett, 1998; Kitayama \& Masuda, 2000; Krull et al., 1999). Of importance, some of these studies have found that the correspondence bias for East Asians is reduced, or even eliminated entirely, when the situational constraints are salient. These same situational salience manipulations have no effect on the correspondence bias shown by Americans, suggesting that the cultural difference in social inference may reflect primarily differences in situational inference. However, these studies were conducted within a single paradigm - attitude attributionand therefore the question remains as to what extent their findings would generalize to other social inference practices, such as behavioral predictions and lay theories of behavior.

\section{Prediction Versus Explanation}

To the extent that Eastern and Western cultures are equally willing to infer dispositions from behavior but differ in the degree to which they apply situational theories to behavior, different implications follow for cultural variation in explanation versus prediction of behavior. In prediction, the outcome of an actor's behavior is not known. As a result, using situational information to predict the behavior of a single individual is difficult because it is unclear to an observer whether the situational influence under consideration applies to that particular individual. Will Tom act aggressively when provoked by an adversary? Even if one believes that provocation is a powerful trigger of violent behavior, not everyone lashes out when provoked, and it is hard to predict in advance if Tom is the sort of person who reacts to provocation. Thus, to the extent that individual differences are perceived in the same situation, the tendency to use situational information is diminished (Ross \& Nisbett, 1991).

The uncertainty characteristic of prediction, however, is greatly reduced in the case of explanation because knowledge of the behavioral outcome provides clues as to whether the situational information applies to the given individual, thus diminishing concerns about individual differences in the same situation. People then can easily apply situational information to the extent that they endorse a situationist theory of behavior. After 
learning that Tom did react violently, one who believes in the power of provocation can conclude with reasonable certainty that provocation led to his aggression. This analysis suggests that cultural variation that readily emerges in explanation may be reduced or even eliminated in prediction, unless salient situational information is available.

To recap, a significant body of evidence has established that there is a cultural difference in situational inference between Western and Eastern cultures, reviewed recently by Choi et al. (1999) (see also Norenzayan, Choi, \& Nisbett, 1999). This evidence, however, has been largely limited to two research paradigms: attitude attribution and causal attribution. There is also suggestive evidence that under some circumstances Easterners display dispositional inference as much as Westerners. However, most of the past research has not examined the distinct contributions of these two processesdispositional and situational- to the cultural difference.

The present research therefore had three goals. First, we broadened the scope of the cross-cultural evidence by examining social inference in two novel research paradigms previously overlooked: behavioral prediction and folk theories of social behavior. Second, these two novel paradigms allowed us to assess the relative contributions of dispositional and situational inference to the cultural difference. Third, we explored the possibility that the East Asian folk theory of personality may be qualitatively different from the Western form in that it may reflect the belief that dispositions are situationally malleable.

We examined (a) the degree to which Koreans, compared to Americans, would rely on individual difference information to predict future behavior when situational information is minimal (Study 1); (b) the effects of situational information of varying salience on Korean and American predictions of the cross-situational consistency of behavior (Study 2); and (c) endorsement of dispositionist, situationist, and interactionist theories of behavior as well as beliefs in the malleability of personality (Studies 1 and 3). Because no gender differences were found in these studies, the results are presented without reference to gender.

\section{STUDY 1}

\section{Overview}

Study 1 had two goals. First, we wanted to answer three specific questions about disposition-based prediction in the absence of situational information. To what extent would East Asians, as compared to Americans, (a) rely on abstract trait information to make predictions about future behavior and (b) rely on information in the form of concrete behaviors-without mention of trait labelsin predicting future behavior (suggesting inference of traits from concrete behavior). We also wanted to investigate the possibility of any cross-cultural differences in the strength of predictions as a function of aggregation of behavioral information, that is, sensitivity to the law of large numbers in social inference.

The second goal of this study was to examine EastWest cultural differences in situational and dispositional attributions using an alternative strategy: We directly measured participants' explicit endorsement of these lay theories of social behavior. We expected that Koreans would be more willing than Americans to endorse situationism and interactionism but that the two cultural groups would be equally willing to endorse dispositionism.

In the first part of the study, we gave participants scenarios; in each, there were two target individuals, and one of them always acted in a trait-consistent manner more than did the other individual, for example, Person A was more helpful than Person B. Participants were asked to predict the extent to which they believed Person A also would behave in a more helpful manner than Person B in a future instance. A total of six traits/behaviors were used: positively valenced (helpful, friendly), negatively valenced (aggressive, dishonest), and relatively valence-neutral (talkative, punctual). In the second part, participants read three arguments representing situationism, dispositionism, and interactionism and indicated their agreement with each argument.

\section{PARTICIPANTS}

Participants included 100 Korean students at Yonsei University, Korea ( 51 men, 49 women, age $M=20.7$ ), and 97 European American students at the University of Michigan (34 men, 63 women, age $M=19$ ) who participated in the study for introductory psychology class credit.

\section{MATERIALS}

The first page of the questionnaire included instructions followed by a rating scale and the six behavioral scenarios. For Korean participants, all materials were translated into Korean using the back-translation method (Brislin, 1970). Each of the six behavioral scenarios consisted of a short paragraph about Person A, who was observed to act in a manner reflecting a trait more than Person B in a given situation. Participants were asked to predict to what extent they believed that in a future, similar situation, A also would act in the trait-consistent way more than B. For the trait condition, participants read the following:

Suppose you observed a number of people in a number of situations that could reflect their degree of (helpfulness). And suppose you observed A and B in one such situation, and A was more (helpful) than B. What do you 
suppose is the probability that if you observed A and B again, you would also find A to be more (helpful) than B?

The rating scale was adopted from Kunda and Nisbett (1986). Participants were asked to indicate any number ranging from $100 \%$ to $50 \%$, with higher numbers indicating greater confidence in the stability of individual differences. ${ }^{1}$ They read the following scale information:

If you thought Person A would almost certainly be more (helpful) than Person B, you should say $95 \%$ or higher. If you thought there was a good chance that Person A would be more (helpful) than Person B, you should say $70 \%$ to $80 \%$. If you thought there was only a moderate chance, you should say $60 \%$ to $70 \%$. And if you thought you couldn't tell whether or not Person A would be more (helpful) than Person B, you should say 50\%-50\%Person A is equally likely to be more or less (helpful) than Person B.

\section{DESIGN AND PROCEDURE}

The design was 2 (cultural group: American vs. Korean) $\times 3$ (behavioral information: concrete behavior, trait, total trait) $\times 3$ (trait valence, within-groups: positive, neutral, negative). Participants were randomly assigned to one of the three behavioral information conditions.

Concrete behavior condition. Participants received individual difference information in the form of concrete behaviors, tapping a trait, but without labeling the behaviors in terms of a trait. An effort was made by American and Korean informants to construct functionally equivalent items. As an example, the concrete behavior scenario reflecting "helpful" was as follows:

Suppose you observed two people, A and B, being asked to participate in a blood donation drive and saw that $\mathrm{A}$ volunteers to work more hours than B collecting blood. What do you suppose is the probability that, being approached by a homeless person asking for money to buy food, A gives more money to the homeless person than B?

Trait condition. Participants received the individual difference information in the form of a trait, such as helpful, dishonest, talkative, and so forth.

Total trait condition. This was identical to the trait condition except that instead of predicting from one single instance to another single instance, participants predicted from the average of 20 instances to the average of another 20 instances. This allowed us to assess sensitivity to the law of large numbers in social prediction.

After making all six predictions, participants read three arguments that had been carefully prepared to reflect three views of social behavior: dispositionism, situationism, and interactionism. They were asked to
TABLE 1: Means and Standard Deviations (in parentheses) of Behavioral Predictions by Culture and Type of Prediction (Study 1)

\begin{tabular}{|c|c|c|c|}
\hline \multirow[b]{2}{*}{ Cultural Group } & \multicolumn{3}{|c|}{ Behavioral Information } \\
\hline & $\begin{array}{c}\text { Concrete Behavior } \\
\mathrm{M}\end{array}$ & $\begin{array}{c}\text { Trait } \\
\mathrm{M}\end{array}$ & $\begin{array}{c}\text { Total Trait } \\
\text { M }\end{array}$ \\
\hline American $(n=97)$ & $71.53(11.35)$ & $70.07(9.53)$ & $77.91(7.23)$ \\
\hline Korean $(n=100)$ & $72.67 \quad(10.66)$ & $75.06(8.32)$ & $74.04(7.41)$ \\
\hline
\end{tabular}

NOTE: The six behaviors/traits were helpful, friendly (positive), talkative, punctual (neutral), aggressive, and dishonest (negative).

express their opinion about each argument on a 9-point scale from 1 (strongly disagree) to 9 (strongly agree). The argument for dispositionism emphasized the consistency and stability of personality and the power of personality in explaining and predicting behavior. In contrast, the argument for situationism stressed the weakness of personality in determining behavior and focused on the power of the situation. The argument for interactionism defended both dispositionism and situationism and highlighted the complex interaction of personality and situation. These arguments can be seen in the appendix.

Results

\section{PREDICTIONS OF BEHAVIOR}

Each participant's prediction estimates for the six scenarios were averaged to serve as the dependent variable $(\alpha=.70)$. The results can be seen in Table 1 . A Culture $\times$ Behavioral Information $\times$ Valence (within-groups) mixed ANOVA was conducted on the dependent variable. There was no main effect of culture, $F<1$. Thus, Americans and Koreans made the same degree of predictions overall. There was a Culture $\times$ Behavioral Information interaction, $F(2,191)=3.94, p<.02$, such that in the trait condition, Korean predictions were stronger than American predictions, $t(64)=2.26, p<.05$, whereas the reverse was the case in the total trait condition, $t(66)=$ $2.18, p<.05$. No cultural differences emerged in the concrete behavior condition, $t<1$. American predictions showed sensitivity to aggregation contrasting the trait condition with the total trait condition, $t(65)=3.80, p<$ .005 , whereas no such sensitivity was evident for Koreans, $t<1$. Neither culture was affected by the trait label manipulation, because there were no differences between the trait condition and the concrete behavior condition, $F<1$, for either contrast.

The results also revealed a main effect of valence, $F(1$, $191)=11.93, p<.005$, such that negative traits received higher predictions than neutral and positive traits. However, this main effect was qualified by culture, $F(1,191)=$ $11.97, p<.005$. Subsequent analyses indicated that this 
pattern did not hold for Korean participants. Whereas Americans reliably gave higher estimates for negative traits than for positive traits, $t(96)=4.77, p<.001$, and neutral traits, $t(96)=2.74, p<.01$, Koreans did not agree with Americans that negative traits are the most crosssituationally stable. Indeed, Korean predictions for negative and positive traits did not differ, $t<1$, although they did agree with Americans that positive traits are less stable than neutral traits, $t(96)=2.81, p<.01$.

\section{FOLK THEORIES OF SOCIAL BEHAVIOR}

For the argument supporting dispositionism, there was no cultural difference; $M=5.00, S D=1.58$ for Americans, and $M=5.19, S D=1.62$ for Koreans, $F<1$. In contrast to dispositionism, Koreans endorsed situationism more than did Americans, $M=5.91, S D=1.42$, and $M=$ $5.43, S D=1.81$, respectively, $F(1,191)=4.44, p<.05$. However, there were no cultural differences for the argument representing interactionism, $M=7.95, S D=1.40$ for Americans, and $M=7.79, S D=1.37$ for Koreans, $F<1$. Interestingly, endorsements of the two arguments supporting dispositionism and situationism were unrelated for both Americans, $r(n=97)=.05, p=n s$, and Koreans, $r(n=100)=.15, p=n s$.

\section{Discussion}

Koreans made predictions as strong as those made by Americans. Presenting the behavioral information in terms of concrete behaviors versus in terms of traits did not change the estimates of Americans or Koreans. This data pattern indicates that in the absence of situational information, Koreans, and perhaps other East Asians as well, can be as likely as Americans to see high cross-situational consistency in social behavior. ${ }^{2}$

In addition, Americans, but not Koreans, showed sensitivity to aggregation of behavior. As to the valence of traits, cultural similarities as well as differences emerged in the way valence affected future predictions. Both cultural samples agreed that positive traits are less stable than (less positive) neutral traits. However, only Americans thought that positive traits are less stable than negative traits, a result consistent with past research (Gidron, Koehler, \& Tversky, 1993).

Moving on to lay theories of social behavior, Americans and Koreans endorsed dispositionism equally, whereas Koreans agreed with situationism more than did the Americans. These data reinforce the findings for predictions of behavior, indicating that East Asians and Westerners are equally willing to see social behavior through the lens of dispositions when situational information is unavailable or nonsalient. The data also show that the two cultures differ reliably in the extent to which situational causes of behavior are highlighted. Finally, it is interesting to note that no cultural differences were found in participants' agreement with the interactionist argument. This is despite evidence that East Asian cultures endorse a contextualist folk psychology, such that behavior is seen as the outcome of complex interactions between the person and the situation (Choi et al., 1999). It is also interesting to note that of the three arguments, the interactionist argument drew the highest agreement ratings on the part of all participants. Thus, both Westerners and Easterners seem to be strong believers in an interactionist view of social behavior.

\section{STUDY 2}

Study 1 demonstrated that Koreans think as dispositionally as Americans in the absence of situational information. These findings support the possibility that the East-West cultural difference in social inference is not to be found in sheer tendency to apply dispositional constructs. The difference thus is more likely to be due to differential sensitivity to salient situational constraints. There are at least two contexts under which this cultural difference could emerge in social prediction.

One possible condition is to have participants make aggregate predictions rather than singular predictions. Aggregate prediction involves predicting the behaviors of a group of people in a given situation rather than the behavior of a single individual in a given situation (e.g., How likely is it that a group of people will be talkative at a party?). In making an aggregate prediction, participants cannot use a dispositional theory of behavior; they have to rely exclusively on their situational theories to make a meaningful prediction. If the source of the East-West cultural difference lies in situationism, then a cultural difference should emerge in aggregate prediction.

A second context in which a cultural difference in situationism should lead to a cultural difference in predicting social behavior is when the situational information is so strong and attention drawing that participants would be compelled to rely on it at least partly despite the availability of conflicting dispositional information. Tom may have been quiet at last month's party but if we learn that at the next party all the guests know and like each other quite well, and you bring it to our attention that most people in this situation become talkative, then it should be hard to ignore this information in our prediction of Tom's behavior. We would expect East Asians to be more likely to apply situational information that is brought to their attention.

In Study 2, therefore, we wanted to achieve three things: First, we measured participants' aggregate predictions-or base rate estimates-in addition to singlecase predictions. Second, participants received strong situational information in addition to dispositional information. Third, we examined the extent to which making the situational information salient-explicitly 
drawing the participants' attention to the situational information-would make them more likely to use that information in behavioral prediction. We examined whether Koreans were more influenced by behavioral base rates in making single-case predictions than Americans.

The situational information was manipulated by asking participants to make behavioral predictions based on information either about a facilitating situation, which made the trait-consistent behavior more likely to occur (e.g., a talkative individual being in a party), or based on an inhibiting situation, which made the traitconsistent behavior less likely to occur (e.g., a talkative individual being at a church service).

We used a priming manipulation to enhance the salience of situational information. Unlike participants in the nonsalient base rate condition, those in the salient base rate condition were asked to make aggregate predictions before answering the single-case prediction questions. We anticipated that answering aggregate predictions (e.g., How many people, out of 100, would do this behavior in this particular situation?) before making predictions about the behavior of a single individual would draw participants' attention to the importance of the situation in influencing the behavior of the target in the single-case prediction task. Thus, the measurement of one dependent variable (the aggregate prediction task) also served as a salience manipulation for the other dependent variable (the single-case prediction task).

Method

\section{PARTICIPANTS}

Study 2 consisted of 58 individuals: 32 students recently arrived from Korea who were learning English at the University of Michigan for the summer period (17 men, 15 women, age $M=22$ ) and 26 American students (25 European American, 1 African American; 11 men, 15 women, age $M=21$ ) recruited from among undergraduates at the university. All participants were paid $\$ 10$ for their participation in the study.

\section{INSTRUCTIONS}

Participants received a six-page questionnaire. For Korean participants, the questionnaire was translated and back-translated (Brislin, 1970) by two bilingual Korean graduate students. In the Korean version of the questionnaire, we used common Korean names to replace the English names and Korean equivalents of American cultural items. As in the previous studies, participants were asked to make predictions about the future behavior of a target individual using the same six behavioral scenarios. Unlike the previous study, however, participants were asked only about one actor who behaved in a trait-consistent manner. ${ }^{3}$

\section{SITUATIONAL INFORMATION MANIPULATION}

Half of the participants received situational information that would facilitate the behavior and the other half received situational information that would inhibit the behavior. The following is the scenario for "helpful" in the inhibiting condition:

\begin{abstract}
You just met a new neighbor, Jim. As you and Jim are taking a walk in the neighborhood, a well-dressed man approaches Jim and explains that his car is broken down and he needs to call a mechanic. Then with a somewhat embarrassed voice the man asks Jim for a quarter to make the phone call. You find that Jim searches his pocket and after finding a quarter gives it to the man. On another day Jim is walking toward the bus stop to catch the bus to work. As he is walking, a teenager carrying some books approaches Jim and politely asks him if he can borrow a dollar for a bus ride, explaining that he forgot his wallet at home and needs to get a ride to school. Jim searches his pocket and discovers that he only has one dollar with him. He realizes that if he gives the dollar to the teenager, he will not be able to ride the bus himself and will miss an important business meeting. Do you think Jim will give the dollar to the teenager?
\end{abstract}

As can be seen in the example above, the dispositional information was given in the first paragraph in the form of a behavior (giving a quarter for a phone call) implying a trait (helpful). The second part of the scenario introduces the situational information, in this case inhibiting information (being helpful would mean missing an important business meeting for the actor).

The facilitating condition was identical to the inhibiting one except that in the second part participants received facilitating information. Thus, instead of having only one dollar in his pocket, Jim "searches his pocket and discovers that he has several dollars with him, enough money to pay for his bus ride and for the teenager's." Furthermore, there was no mention of an important business meeting in the facilitating scenario.

\section{DEPENDENT MEASURES}

For single-case predictions, participants read the following in the instructions:

\footnotetext{
Your answer can be any number between 0 and 100 . Zero indicates that you are absolutely certain that the person will not do the behavior in the future. One hundred indicates that you are absolutely certain that the person will do that behavior in the future.
}

For the aggregate prediction task, participants were instructed to estimate what proportion of 100 randomly selected individuals would do each behavior in the situation being described. The aggregate prediction scenarios were identical to the single-case prediction scenarios 
except that the dispositional information was removed: Participants read only the second part of the prediction scenarios containing the situational information (inhibiting vs. facilitating). Thus, participants in the salient base rate condition first made aggregate predictions (i.e., generated base rate estimates) for each situation, then read the dispositional and situational information about a single target person, and then predicted the target's behavior. Participants in the nonsalient base rate condition first read dispositional and situational information about the target, predicted the target's future behavior, and then proceeded to make aggregate predictions.

\section{DESIGN OF STUDY}

The design was 2 (cultural group: American vs. Korean) $\times 2$ (situational information: facilitating vs. inhibiting) $\times 2$ (salience: aggregate predictions before vs. after single-case predictions), all between-subjects conditions.

Results

Aggregate predictions and singular predictions were averaged across the six scenarios to produce a single average aggregate prediction score and a single average single-case prediction score for each participant, with respective internal reliability coefficients of $\alpha=.83$ and $\alpha=.88$.

\section{AGGREGATE PREDICTIONS}

Figure 1 shows the aggregate predictions. A 2 (cultural group: American vs. Korean) $\times 2$ (situational information: facilitating vs. inhibiting) $\times 2$ (aggregate predictions before vs. after single-case predictions) betweensubjects ANOVA on aggregate predictions revealed a large main effect of situational information, such that inhibiting situations received lower aggregate predictions compared to facilitating situations, $F(1,50)=$ 408.40, $p<.001$. As expected, a Culture $\times$ Situational Information interaction emerged, such that Korean aggregate predictions were more responsive to the situational information than American predictions, $F(1,50)=$ $7.71, p<.01$; Korean predictions were higher when the situation was facilitating and lower when the situation was inhibiting.

\section{SINGLE-CASE PREDICTIONS}

Figure 2 shows the single-case predictions for each cultural group. A Culture (American vs. Korean) $\times$ Situational Information (facilitating vs. inhibiting) $\times$ Salience (salient base rate vs. nonsalient base rate) ANOVA conducted on the single-case predictions again revealed a large main effect of situational information, with inhibiting scenarios receiving lower prediction estimates compared to facilitating scenarios, $F(1,50)=256.46, p<.001$,

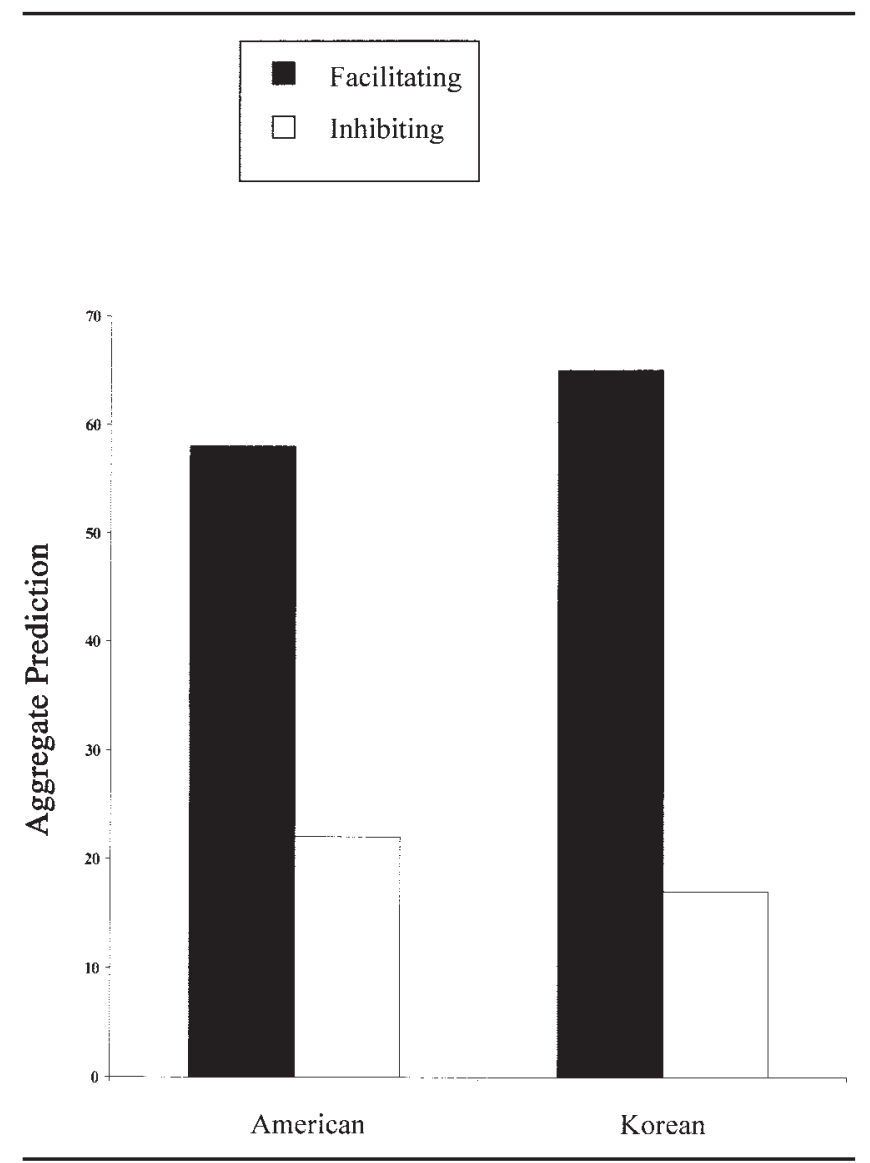

Figure 1 Mean aggregate predictions for each cultural group and type of situational information (facilitating vs. inhibiting) averaged across six behaviors.

NOTE: Means indicate the estimated number of people out of a randomly selected 100 who would engage in the behavior specified in each scenario (Study 2).

$M=30.08$ and $M=78.07$. Also, a Situational Information $\times$ Salience interaction was observed, $F(1,50)=10.93, p<$ .005 . However, this two-way interaction will not be discussed further because a three-way interaction between culture, situational information, and salience of situational information emerged, $F(1,50)=4.60, p<.05$. This finding indicates that Koreans were more likely than Americans to apply situational information to predict the behavior of the target individual, but only when the situational information was made salient by having participants make aggregate predictions before making single-case predictions. There were no differences between American and Korean predictions when base rate predictions came after.

The cultural difference in sensitivity to situational constraints is especially apparent when the situational salience manipulation is considered for each cultural group. For Koreans, single-case predictions in the salient base rate condition were more responsive to the base rates than the predictions in the nonsalient base rate 


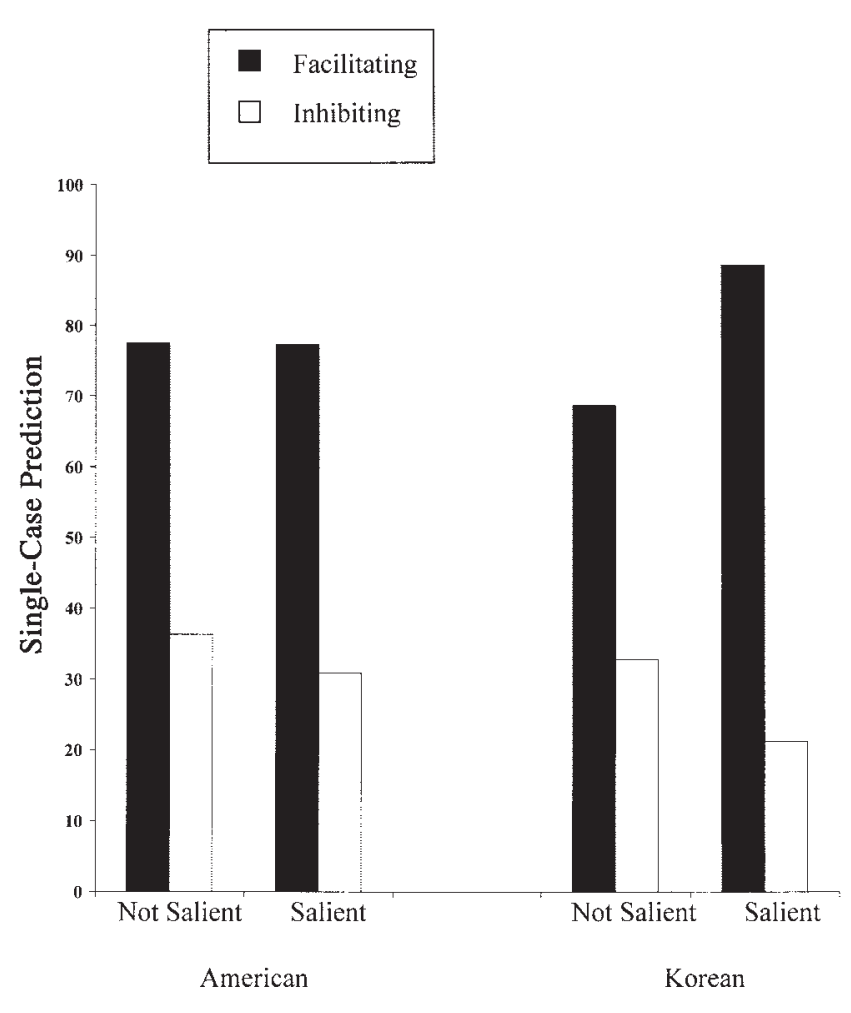

Figure 2 Mean single-case predictions for each cultural group, type of situational information (facilitating vs. inhibiting), and salience of situational information averaged across all six behaviors.

NOTE: Higher numbers indicate stronger predictions for future behavior (Study 2).

condition, $t(50)=3.50, p<.005$, for facilitating situations, and $t(50)=2.01, p<.05$, for inhibiting situations. In contrast, American predictions were not affected at all by the salience manipulation, $t<1$, for both facilitating and inhibiting situations.

CULTURAL DIFFERENCE IN SINGLE-CASE

PREDICTION OR IN CONVERSATIONAL LOGIC?

We now consider an alternative conversational logic or experimental demand interpretation for the cultural difference in the single-case predictions. Asking people to make aggregate predictions before making singlecase judgments may have created a conversational implicature, or an experimental demand, to use the outcome of the prior judgment in the subsequent one. Moreover, if Easterners are more attuned to social cues from other people, they might be especially sensitive to informational cues provided by the researcher.

Despite the plausibility of this argument, there is no reported evidence indicating that Easterners are actually more responsive to experimental cues than are Westerners. In fact, there is some evidence that Easterners can be less responsive to experimental instructions than Westerners (e.g., Choi \& Nisbett, 1998, Study 2). Nevertheless, if Easterners were more sensitive to experimental cues in this study, they would be expected to use the base rate information in the subsequent single-case predictions more than Westerners.

According to this interpretation, then, Koreans may have been more compelled to use any prior information that preceded a task, rather than being more compelled to apply situational information per se. This alternative interpretation can be tested in the condition when the single-case judgment preceded the aggregate judgment. This interpretation predicts that Koreans should have been more compelled to apply the dispositional information (contained in the single-case prediction task; e.g., a helpful behavior) to the subsequent aggregate judgment. That is, Koreans should have used the individual helpful act (in single-case prediction) more to estimate higher rates of helpfulness by a group of 100 individuals (in aggregate prediction), for both the facilitating and inhibiting scenarios.

However, this was not the case. There was no main effect of order of the two tasks on aggregate predictions, $F(1,50)=1.40, p=n s$, and most important, no two-way interaction between culture and order, $F(1,50)=1.12$, $p=n s$, or a three-way interaction between culture, type of situational information, and order, $F<1$. Exposure to the dispositional information in the single-case prediction task affected neither Americans' nor Koreans' subsequent aggregate predictions. Thus, there is some evidence that the Koreans' greater use of self-generated base rates in single-case prediction was not due to a general tendency to make greater use of any preceding information presented by the experimenter. Rather, it was due to a greater Korean tendency to use specifically situational information in social judgment.

\section{Discussion}

The main effect of situational information on aggregate predictions as well as on single-case predictions indicates that both American and Korean participants can be sensitive to sharp variations in situational information. The interesting finding, however, is that Korean aggregate predictions were more sensitive to base rates than those of Americans.

As for the single-case predictions, when dispositional theories were countered by salient situational information, Korean participants were more likely than Americans to apply their situationist beliefs in predicting social behavior. Furthermore, increasing the salience of situational base rates influenced Korean predictions in the expected manner but had no effect on American predictions. This pattern reinforces findings obtained by Choi 
and Nisbett (1998) and Kitayama and Masuda (2000) in the Jones and Harris (1967) attitude attribution paradigm. When situational constraints were made salient to the participants, Koreans and Japanese corrected their correspondent inferences. The same situational salience manipulation had no effect on American attributions (Choi \& Nisbett, 1998; Snyder \& Jones, 1974; but see Newman \& Ruble, 1992, for evidence that American children correct their correspondence inferences when situational constraints are salient).

\section{STUDY 3}

Study 3 was conducted to replicate and further extend the findings of Study 1. Participants read again the three arguments of Study 1 and expressed their degree of agreement with them. Furthermore, we examined the possibility that the nature of the dispositional theories held by the two cultures is different, even if Easterners and Westerners are both likely to engage in dispositional inference. The coexistence of situationism and dispositionism in Eastern folk psychology should lead Easterners to have theories indicating that dispositions are more malleable and more likely to incorporate situational variability than are theories of Westerners. Thus, Western dispositionism might be analogous to what Dweck and her colleagues call "entity theory," whereas East Asian dispositionism may be more like "incremental theory" (Dweck, Hong, \& Chiu, 1993). Both theories are about dispositions, but they differ regarding their malleability. Entity theorists believe that dispositions such as personality, intelligence, and moral character are fixed and that people cannot change them at will. In contrast, incremental theorists believe that dispositions are flexible and malleable. Dweck and her colleagues demonstrate that social judgment differs depending on the implicit theory a person has. Compared to entity theorists, incremental theorists make trait inferences that are more specific (in that they do not endorse global traits), more provisional (in that they are responsive to contradictory information), and more conditional (in that they expect change with changed circumstances).

To explore the possibility that East Asians are indeed more like incremental theorists, whereas Americans are more like entity theorists, Study 3 measured implicit theory of personality (entity vs. incremental theory). In addition, we measured endorsement of lay theories of social behavior. We expected no difference in dispositionist theory of behavior and a cultural divergence in situationist and possibly interactionist theories of behavior.
TABLE 2: Means and Standard Deviations (in parentheses) of Disagreement With Statements Representing Entity Theory of Personality (Study 3 )

\begin{tabular}{llll}
\hline & American & Korean & $\mathrm{p}$ \\
\hline $\begin{array}{l}\text { Someone's personality is } \\
\text { something about them that } \\
\text { they can't change very much. }\end{array}$ & $3.50(1.27)$ & $4.04(1.28)<.001$ \\
$\begin{array}{l}\text { A person can do things to get } \\
\text { people to like them but they }\end{array}$ & & & \\
$\quad \begin{array}{l}\text { can't change their real } \\
\text { personality. }\end{array}$ & $3.09(1.30)$ & $3.79(1.37)<.001$ \\
$\begin{array}{l}\text { Everyone has a certain personality } \\
\text { and it is something that they }\end{array}$ & & & \\
$\quad \begin{array}{l}\text { can't do much about. } \\
\text { A person can change the way } \\
\text { they act but they can't change } \\
\text { their real personality. }\end{array}$ & $3.41(1.28)$ & $3.99(1.31)<.001$ \\
\hline
\end{tabular}

NOTE: Higher numbers indicate stronger disagreement with entity theory of personality on a 6-point scale.

\section{Method}

\section{PARTICIPANTS}

Korean students at So-Gang University in Korea $(N=$ 120) and American students at the University of Michigan $(N=121)$ took part in the study for introductory psychology class credit.

\section{PROCEDURE}

For Korean participants, all materials were translated into Korean using the back-translation method (Brislin, 1970). Participants were given a booklet containing the same three arguments of Study 3 reflecting dispositionism, situationism, and interactionism (see Table 1). They were asked to express their opinion about each argument on a 9-point scale from 1 (strongly disagree) to 9 (strongly agree).

Participants then read four statements devised by Erdley and Dweck (1993) intended to measure implicit theory of personality. They were asked to express their agreement with each statement on a 6-point scale from 1 (strongly agree) to 6 (strongly disagree). The four statements can be seen in Table 2. Because incremental theory of personality elicits socially desirable responding, endorsement of an incremental view of personality is measured in terms of disagreement with statements that reflect an entity theory of personality (Chiu, Hong, \& Dweck, 1997). Extensive research supports the validity of this strategy. For example, when asked to justify their responses, people who disagree with entity theory provide explanations that are consistent with an incremental view of personality, and participants who are experimentally induced to think in a manner consistent with 
incremental theory exhibit behavior that is similar to those participants who disagree with entity statements (see Chiu et al., 1997, for a review of the literature validating the measurement of incremental theory in terms of disagreement with entity theory). Thus, greater disagreement with these "entity" statements indicates greater endorsement of incremental theory of personality, although incremental theory is not measured directly in this study.

Results

\section{FOLK THEORIES OF SOCIAL BEHAVIOR}

For the argument supporting dispositionism, there was no cultural difference; $M=4.59, S D=1.79$ for Americans, and $M=4.82, S D=1.79$ for Koreans, $F<1$. However, Korean participants agreed with the argument representing situationism more than American participants did, $M=5.27, S D=1.46$, and $M=4.67, S D=1.62$, respectively, $F(1,240)=8.47, p<.005$. Unlike in Study 3, the same cultural difference this time emerged also for the argument representing interactionism, $M=7.32, S D=$ 2.01 for Americans, and $M=7.99, S D=.98$ for Koreans, $F(1,240)=10.63, p<.001$.

\section{FOLK THEORIES OF PERSONALITY}

We averaged participants' ratings of the four arguments and compared the two cultures $(\alpha=.91$ and .92 for Americans and Koreans, respectively). As expected, Koreans disagreed with entity theory more than Americans did $(M=3.87, S D=1.21$ for Koreans, and $M=3.27$, $S D=1.14$ for Americans $), F(1,240)=15.60, p<.001$. Indeed, as can be seen in Table 2, Koreans disagreed with all four statements more than Americans did.

\section{Discussion}

The data on lay theory of social behavior in this study support our findings in the previous studies: East Asians and Westerners differed reliably in situationism but not in dispositionism. We also found that East Asians endorsed interactionism more than Americans did. This, we believe, is also a reflection of East Asians' greater sensitivity to situational influence. Thus, participants' theory of social behavior converges on the behavioral prediction data reported in Study 1. Interestingly, the interactionist argument again drew the highest rate of endorsement, indicating that it is the theory that both cultures endorse the most.

The findings on lay theory of personality indicate that personality theories in East and West differ in an important way: Easterners believe in the malleability of dispositions more than Americans do. Thus, Korean participants' thinking was more like that of incremental theorists of personality, whereas American participants' thinking was more like that of entity theorists.

\section{GENERAL DISCUSSION}

Study 1 showed that when situational information was absent, Koreans and Americans drew the same dispositional inferences about an actor's behavior and thus predicted equally high behavioral consistency across situations. In Study 2, Koreans made predictions about aggregate behavior that were more influenced by situational information than those of Americans. Also, when predicting the behavior of a single individual, highly accessible situational information affected Korean but not American predictions. Finally, whereas Koreans and Americans were equally likely to endorse a dispositionist theory of behavior, Koreans agreed with a situationist theory more (Studies 1 and 3 ).

The present research indicates that cultural differences in social inference are not confined to causal explanation and attitude attribution but operate for predictions of behavior and lay theories of behavior as well. Thus, cultural differences in social inference permeate a broad range of inferential practices. However, cultural variation in social inference is context sensitive. It is robust under conditions when situational information is highly accessible and decreases and may disappear entirely to the extent that situational information is relatively difficult to apply.

Even though East Asians are willing to infer dispositions from behavior as much as Westerners, Eastern and Western dispositionism are not qualitatively identical. Easterners believe in the continuous shaping of personality traits by situational influences. This Eastern belief in the alterability of personality traits parallels the distinction between people who subscribe to entity theory versus incremental theory of personality (e.g., Dweck et al., 1993). We provided preliminary evidence that Western dispositionism is like entity theory, in that it reflects the belief that personality traits are fixed. In contrast, Eastern dispositionism is more like incremental theory, in that it reflects the belief that personality traits are malleable (Study 3).

\section{Lay Dispositionism as Psychological Essentialism}

Why should lay dispositionism be more resistant to cross-cultural variation than previously anticipated? A possible theoretical explanation may be that lay dispositionism is a form of psychological essentialism, a mode of thinking that consists of attributing a hidden essence or quality to a thing, which causes its observable as well as hidden properties (Atran, 1990; Gelman, 1992; Gelman \& Hirschfeld, 1999). Essentialism has been observed in a number of diverse domains, notably in reasoning about animals and social groups in a large variety of cultures (Gelman \& Hirschfeld, 1999). Gelman (1992) observes that folk biological and dispositional thinking are strikingly similar, raising the possibility that 
dispositionism is essentialism in the domain of folk psychology. Just as the "biological essence" of a zebra causes it to develop a striped fur and act like a zebra, an individual's personality, or "psychological essence," causes a person to think, feel, and behave in particular ways.

It is likely that both Easterners and Westerners essentialize personality relative to other domains that do not support essentialistic reasoning, such as artifacts. Future research can establish the relative strength of essentialism in personality theories across cultures. Our data, indicating that Easterners view personality as more malleable than Westerners, suggests the possibility that cultures that endorse situationism may be less likely to essentialize personality.

\section{Cultural Variation in Social Inference}

The evidence we presented suggests that what varies across cultures is the situational inference part of the attribution process, whereas dispositional inference is less susceptible to cultural variation. Our studies support other cross-cultural investigations, which have reported a similar pattern of findings. For example, in a Korean replication of the classic McArthur (1972) study, Cha and Nam (1985) found that Korean attributions relied substantially on consensus information, indicating greater East Asian sensitivity to situational information. However, Koreans did not differ from Americans in response to distinctiveness and consistency information. Thus, Koreans were as likely as Americans to use dispositional information.

Because East Asians are willing to apply their personality theories in social judgment when the situational information is absent or nonobvious, they can be as prone to the correspondence bias as Americans. This has been illustrated in recent cross-cultural replications of the correspondence bias in Jones and Harris's (1967) attitude attribution paradigm for Koreans (Choi \& Nisbett, 1998), Japanese (Kitayama \& Masuda, 2000), and Chinese (Krull et al., 1999). However, when Choi and Nisbett (1998) and Kitayama and Masuda (2000) made the situational constraints obvious to the participants, both Koreans and Japanese showed very little correspondence bias. The same manipulation had no effect on the correspondence bias for American samples (Choi \& Nisbett, 1998; Snyder \& Jones, 1974).

Finally, identifying the attributional source and boundary conditions of the cultural variation in social inference has implications for the psychology of cultural misunderstandings. The evidence suggests that when situational information about the other is absent, both cultures will attribute behavior to its corresponding disposition; thus, no cultural misunderstanding will occur that reflects attributional differences. But when salient situational information is available to observers, cultural mis- understanding can emerge. In such situations, East Asians will be attuned to situational information to a greater extent than Westerners and will be more likely to attribute the other's behavior to situational constraints. Research that illuminates these processes can tell us much about the social psychological roots of intercultural misunderstandings.

\section{APPENDIX \\ Lay Theories of Social Behavior \\ Presented to Participants (Studies 1 and 3)}

\section{Dispositionism}

How people behave is mostly determined by their personality. One's personality predisposes and guides an individual to behave in one way, not in another way, no matter what circumstances the person is in. In a sense, behavior is an unfolding of personality. One's behavior is remarkably stable across time and consistent across situations because it is guided by personality. Therefore, if we know the personality of one person, we can easily predict how the person will behave in the future and explain why that person behaved in the particular way in the past.

\section{Situationism}

How people behave is mostly determined by the situation in which they find themselves. Situational power is so strong that we can say it has more influence on behavior than one's personality. Often, people in a particular situation behave very similarly, despite large individual differences in personality. Therefore, in order to predict and explain one's behavior, we have to focus on the situation rather than personality. Personality plays a weaker role in behavior than we used to think.

\section{Interactionism}

How people behave is always jointly determined by their personality and the situation in which they find themselves. We cannot claim that either personality or the situation is the only determinant of our behavior. Our behavior is an outcome of the complex interaction between personality and situational factors. We always have to consider personality and situation simultaneously. Therefore, we cannot predict and explain one's behavior with personality or situation alone.

\section{NOTES}

1. It should be noted, however, that this scale restricts the range of possible responses. Although it is unlikely, one may believe that once Person A engages in a behavior more than Person B, the subsequent chance of A exhibiting the same behavior will be less than for $\mathrm{B}$. This scale prevents participants from expressing this belief adequately. However, this restriction in itself does not bias the scale toward systematic overestimation. When lay estimates are compared with actual estimates using this scale, domains other than personality traits produce moderate accuracy for abilities such as athletics, very high accuracy for student course evaluations, and systematic underestimation in other instances, for example, ability estimates made by professional psychologists (Kunda \& Nisbett, 1986). Moreover, this restriction does not 
interfere with cross-cultural comparisons because the same restriction exists across cultures.

2. This finding of no cultural differences in behavioral predictions was replicated with a sample of Chinese participants. Chinese students from Taiwan at a large American university $(n=41)$, and a different group of American university students at the same institution $(n=98)$, completed a questionnaire very similar to the one used in Study 1. Results were largely consistent to the ones reported here. No main effect of culture was obtained, $F(1,133)<1$, and there was no culture by behavioral information interaction, $F(2,133)<1$. There was a significant effect of aggregation for both cultures.

3. This methodological change was necessary because it was not meaningful to introduce strong situational constraint information while contrasting the behavior of two actors. For a strong situational constraint to be meaningful, it requires that individual differences be relatively slight.

\section{REFERENCES}

Atran, S. (1990). Cognitive foundations of natural history. New York: Cambridge University Press.

Brislin, R. W. (1970). Back-translation for cross-cultural research. Journal of Cross-Cultural Psychology, 1, 185-216.

Cha, J. H., \& Nam, K. D. (1985). A test of Kelley's cube theory of attribution: A cross-cultural replication of McArthur's study. Korean Social Science Journal, 12, 151-180.

Chiu, C., Hong, Y., \& Dweck, C. S. (1997). Lay dispositionism and implicit theories of personality. Journal of Personality and Social Psychology, 73, 19-30.

Choi, I., \& Nisbett, R. E. (1998). Situational salience and cultural differences in the correspondence bias and the actor-observer bias. Personality and Social Psychology Bulletin, 24, 949-960.

Choi, I., Nisbett, R. E., \& Norenzayan, A. (1999). Causal attribution across cultures: Variation and universality. Psychological Bulletin, 125, 47-63.

Dunning, D., Griffin, D. W., Milojkovic, J., \& Ross, L. (1990). The overconfidence effect in social prediction. Journal of Personality and Social Psychology, 58, 588-581.

Dweck, C. S., Hong, Y., \& Chiu, C. (1993). Implicit theories: Individual differences in the likelihood and meaning of dispositional inference. Personality and Social Psychology Bulletin, 19, 644-656.

Erdley, C. A., \& Dweck, C. S. (1993). Children's implicit personality theories as predictors of their social judgments. Child Development, $64,863-878$

Fiske, A. P., Kitayama, S., Markus, H. R., \& Nisbett, R. E. (1998). The cultural matrix of social psychology. In D. T. Gilbert, S. T. Fiske, \& G. Lindzey (Eds.), Handbook of social psychology (4th ed., pp. 915981). New York: McGraw-Hill.

Gelman, S. A. (1992). Children's conception of personality traits: Commentary. Human Development, 35, 280-285.

Gelman, S. A., \& Hirschfeld, L. (1999). How biological is essentialism? In D. A. Medin \& S. Atran (Eds.), Folkbiology (pp. 403446). Cambridge, MA: MIT Press.

Gidron, D., Koehler, D. J., \& Tversky, A (1993). Implicit quantification of personality traits. Personality E Social Psychology Bulletin, 19, 594-604.
Gilbert, D. T., \& Malone, P. S. (1995). The correspondence bias. Psychological Bulletin, 117, 21-38.

Heider, F. (1958). The psychology of interpersonal relations. New York: John Wiley.

Hirschfeld, L. A. (1995). Anthropology, psychology, and the meanings of social causality. In D. Sperber, D. Premack, \& A. Premack (Eds.), Causal cognition: A multidisciplinary debate (pp. 313-350). Oxford, UK: Oxford University Press.

Jones, E. E. (1979). The rocky road from acts to dispositions. American Psychologist, 3, 107-117.

Jones, E. E., \& Harris, V. A. (1967). The attribution of attitudes. Journal of Experimental Social Psychology, 3, 1-24.

Kashima, Y., Siegal, M., Tanaka, K., \& Kashima, E. S. (1992). Do people believe behaviors are consistent with attitudes? Towards a cultural psychology of attribution processes. British Journal of Social Psychology, 31, 111-124.

Kitayama, S., \& Masuda, T. (2000). Cultural psychology of social inference: The correspondence bias in Japan. Unpublished manuscript, Kyoto University.

Krull, D. S. (1993). Does the grist change the mill? The effect of the perceiver's inferential goal on the process of social inference. Personality and Social Psychology Bulletin, 19, 340-348.

Krull, D. S., Loy, M. H., Lin, J., Wang, C., Chen, S., \& Zhao, X. (1999). The fundamental fundamental attribution error: Correspondence bias in individualist and collectivist cultures. Personality and Social Psychology Bulletin, 25, 1208-1219.

Kunda, Z., \& Nisbett, R. E. (1986). The psychometrics of everydaylife. Cognitive Psychology, 18, 195-224.

McArthur, L. Z. (1972). The how and what of why: Some determinants and consequences of causal attribution. Journal of Personality and Social Psychology, 13, 733-742.

McCrae, R. R., Costa, P. T., \& Yik, S. M. (1996). Universal aspects of Chinese personality structure. In M. H. Bond (Ed.), The handbook of Chinese psychology (pp. 189-207). New York: Oxford University Press.

Newman, L. (1996). Trait impressions as heuristics for predicting future behavior. Personality and Social Psychology Bulletin, 22, 395411.

Newman, L., \& Ruble, D. N. (1992). Do young children use the discounting principle? Journal of Experimental Social Psychology, 28, $572-593$

Norenzayan, A., Choi, I., \& Nisbett, R. E. (1999). Eastern and Western perceptions of causality for social behavior: Lay theories about personalities and situations. In D. A. Prentice \& D. T. Miller (Eds.), Cultural divides (pp. 239-272). New York: Russell Sage Foundation.

Ross, L., \& Nisbett, R. E. (1991). The person and the situation: Perspectives of social psychology. New York: McGraw-Hill.

Snyder, M., \& Jones, E. E. (1974). Attitude attribution when behavior is constrained. Journal of Experimental Social Psychology, 10, 585-600.

Received October 25, 2000

Revision accepted February 22, 2001 